Proyecciones Journal of Mathematics

Vol. 34, No 2, pp. 127-136, June 2015.

Universidad Católica del Norte

Antofagasta - Chile

\title{
A note on complementary tree domination number of a tree
}

\author{
B. Krishnakumari \\ SASTRA University, India \\ and \\ Y. B. Venkatakrishnan \\ SASTRA University, India \\ Received: August 2014. Accepted : April 2015
}

\begin{abstract}
A complementary tree dominating set of a graph $G$, is a set $D$ of vertices of $G$ such that $D$ is a dominating set and the induced sub graph $\langle V \backslash D\rangle$ is a tree. The complementary tree domination number of a graph $G$, denoted by $\gamma_{c t d}(G)$, is the minimum cardinality of a complementary tree dominating set of $G$. An edge-vertex dominating set of a graph $G$ is a set $D$ of edges of $G$ such that every vertex of $G$ is incident with an edge of $D$ or incident with an edge adjacent to an edge of $D$. The edge-vertex domination number of a graph, denoted by $\gamma_{e v}(G)$, is the minimum cardinality of an edge-vertex dominating set of $G$. We characterize trees for which $\gamma(T)=\gamma_{c t d}(T)$ and $\gamma_{c t d}(T)=\gamma_{e v}(T)+1$.
\end{abstract}

Keywords : Dominating set; Complementary tree dominating set; edge-vertex dominating set; tree.

AMS Subject Classification : 05C69. 


\section{Introduction}

Let $G=(V, E)$ be a graph. By the neighborhood of a vertex $v$ of $G$ we mean the set $N_{G}(v)=\{u \in V(G): u v \in E(G)\}$. The degree of a vertex $v$, denoted by $d_{G}(v)$, is the cardinality of its neighborhood. By a leaf we mean a vertex of degree one, while a support vertex is a vertex adjacent to a leaf. We say that a support vertex is strong (weak, respectively) if it is adjacent to at least two leaves (exactly one leaf, respectively). We denote by $P_{n}$ the path on $n$ vertices. Let $T$ be a tree, and let $v$ be a vertex of $T$. We say that $v$ is adjacent to a path $P_{n}$ if there is a neighbor of $v$, say $x$, such that the subtree resulting from $T$ by removing the edge $v x$ and which contains the vertex $x$ as a leaf, is a path $P_{n}$. By a star we mean a connected graph in which exactly one vertex has degree greater than one.

A subset $D \subseteq V(G)$ is a dominating set of $G$ if every vertex of $V(G) \backslash D$ has a neighbor in $D$. The domination number of $G$ is the minimum cardinality of a dominating set of $G$. For a comprehensive survey of domination in graphs, the reader is referred to $[1,2]$.

A set $D \subset V(G)$ is a complementary tree dominating set, abbreviated CTDS, of $G$ if it is a dominating set and the induced sub graph $\langle V \backslash D\rangle$ is a tree. The complementary tree domination number of $G$, denoted by $\gamma_{c t d}(G)$, is the minimum cardinality of a complementary tree dominating set of $G$. A complementary tree dominating set of $G$ of minimum cardinality is called a $\gamma_{c t d}(G)$-set. Complementary tree domination was introduced and studied in [5].

An edge $e \in E(G)$ dominates a vertex $v \in V(G)$ if $e$ is incident with $v$ or $e$ is incident with a vertex adjacent to $v$. A subset $D \subseteq E(G)$ is an edge vertex dominating set, abbreviated EVDS, of a graph $G$ if every vertex of $G$ is edge vertex dominated by an edge of $D$. The edge vertex domination number of a graph $G$, denoted by $\gamma_{e v}(G)$, is the minimum cardinality of an edge vertex dominating set of $G$. Edge vertex domination in graphs was introduced in $[6]$.

Trees with equal domination and total domination numbers are characterized in [3] and in [4], trees with double domination number equal to 2-domination number plus one were characterized. We characterize all trees with equal domination and complementary tree domination numbers and in section 3, we characterize all trees with complementary tree domination number equal to edge-vertex domination number plus one. 


\section{Trees with equal domination and complementary tree dom- ination numbers}

In [5] we can find out the following elementary but useful observations.

Observation 1. Every leaf is in every $\gamma_{c t d}(T)$-set.

Observation 2. For every graph $G$ we have $\gamma(G) \leq \gamma_{c t d}(G)$.

We characterize all trees with equal domination and complementary tree domination numbers. For this purpose we introduce a family $T$ of trees $T=T_{k}$ that can be obtained as follows. Let $T_{1}$ be a path $P_{4}$. If $k$ is a positive integer, then $T_{k+1}$ can be obtained recursively from $T_{k}$ by one of the following operations.

- Operation $O_{1}$ : Attach a path $P_{2}$ by joining its any vertex to a vertex of $T_{k}$, which is not a leaf and is adjacent to a support vertex of degree two.

- Operation $\mathrm{O}_{2}$ : Attach a path $P_{2}$ by joining its any vertex to a support vertex of $T_{k}$.

We now prove that for every tree $T$ of the family $T$, the domination and the complementary tree domination numbers are equal.

Lemma 1. If $T \in T$, then $\gamma_{c t d}(T)=\gamma(T)$.

Proof. We use the induction on the number $k$ of operations performed to construct the tree $T$. If $T=T_{1}=P_{4}$, then $\gamma_{c t d}(T)=2=\gamma(T)$. Let $k \geq 2$ be an integer. Assume the result is true for every tree $T^{\prime}=T_{k}$ of the family $T$ constructed by $k-1$ operations. Let $T=T_{k+1}$ be a tree of the family $T$ constructed by $k$ operations.

First assume that $T$ is obtained from $T^{\prime}$ by operation $O_{1}$. The vertex to which is attached $P_{2}$ we denote by $x$. Let $u_{1} u_{2}$ be the attached path. Let $u_{1}$ be joined to $x$. Let $y$ be a support vertex of degree two adjacent to $x$ and different from $u_{1}$. Let $z$ be the leaf adjacent to $y$. Let $D^{\prime}$ be a $\gamma_{c t d}\left(T^{\prime}\right)$-set. By observation 1, the leaf $z \in D^{\prime}$. Either $y \in D^{\prime}$ and $N[x] \backslash\{y\} \notin D^{\prime}$ or $N(x) \backslash\{y\} \in D^{\prime}$. In either case it is easy to see that $D^{\prime} \cup\left\{u_{2}\right\}$ is a CTDS of the tree $T$. Thus $\gamma_{c t d}(T) \leq \gamma_{c t d}\left(T^{\prime}\right)+1$. Now let $D$ be a $\gamma(T)$-set. To dominate $u_{2}, z$, the vertex $u_{1}, y \in D$. It is obvious that $D \backslash\left\{u_{2}\right\}$ is a DS of the tree $T^{\prime}$. Thus $\gamma\left(T^{\prime}\right) \leq \gamma(T)-1$. Now we 
get $\gamma_{c t d}(T) \leq \gamma_{c t d}\left(T^{\prime}\right)+1=\gamma\left(T^{\prime}\right)+1 \leq \gamma(T)$. By observation 2 we have $\gamma_{c t d}(T) \geq \gamma(T)$. This implies that $\gamma_{c t d}(T) \geq \gamma(T)$.

Assume that $T$ is obtained from $T^{\prime}$ by operation $O_{2}$. The vertex to which is attached $P_{2}$ we denote by $x$. Let $y$ be the leaf adjacent to $x$. Let $v_{1} v_{2}$ be the attached path. Let $v_{1}$ be joined to $x$. Let $D^{\prime}$ be a $\gamma_{c t d}\left(T^{\prime}\right)$ set. By observation 1, the vertex $y \in D^{\prime}$. It is clear that $D^{\prime} \cup\left\{v_{2}\right\}$ is a CTDS of the tree $T$. Thus $\gamma_{c t d}(T) \leq \gamma_{c t d}\left(T^{\prime}\right)+1$. Now let $D$ be a $\gamma(T)$-set. To dominate $v_{2}, y$, the vertex $v_{1}, x \in D$. It is obvious that $D \backslash\left\{v_{1}\right\}$ is a DS of the tree $T^{\prime}$. Thus $\gamma\left(T^{\prime}\right) \leq \gamma(T)-1$. Now we get $\gamma_{c t d}(T) \leq \gamma_{c t d}\left(T^{\prime}\right)+1=\gamma\left(T^{\prime}\right)+1 \leq \gamma(T)$. By observation 2 we have $\gamma_{c t d}(T) \geq \gamma(T)$. This implies that $\gamma_{c t d}(T) \geq \gamma(T)$.

We now prove that if the domination and complementary tree domination numbers of a tree are equal, then the tree belongs to the family $T$.

Lemma 2. Let $T$ be a tree. If $\gamma_{c t d}(T)=\gamma(T)$, then $T \in T$.

Proof. Let $n$ mean the number of vertices of the tree $T$. We proceed by induction on this number. If $\operatorname{diam}(T)=1$, then $T=P_{2}$. We have $\gamma_{c t d}(T)=1=\gamma(T)$. Now assume that $\operatorname{diam}(T)=2$. Thus $T$ is a star. We have $\gamma_{c t d}(T)=n-1>1=\gamma(T)$.

Now assume that $\operatorname{diam}(T) \geq 3$. Thus the order $n$ of the tree $T$ is at least four. We obtain the result by the induction on the number $n$. Assume that the theorem is true for every tree $T^{\prime}$ or order $n^{\prime}<n$.

First assume that some support vertex of $T$, say $x$ is strong. Let $y, z$ be a leaf adjacent to $x$. Let $T^{\prime}=T-y$. Let $D$ be a $\gamma_{c t d}(T)$-set. By observation 1 , the leaf $y, z \in D$. It is obvious that $D \backslash\{y\}$ is a CTDS of the tree $T^{\prime}$. Thus $\gamma_{c t d}\left(T^{\prime}\right) \leq \gamma_{c t d}(T)-1$. Let $D^{\prime}$ be a $\gamma\left(T^{\prime}\right)$-set. To dominate $z$, the vertex $x \in D$. It is clear that $D^{\prime}$ is a DS of the tree $T$. Thus $\gamma(T) \leq \gamma\left(T^{\prime}\right)$. We get $\gamma_{c t d}\left(T^{\prime}\right) \leq \gamma_{c t d}(T)-1=\gamma(T)-1 \leq \gamma\left(T^{\prime}\right)-1<\gamma\left(T^{\prime}\right)$.

We now root $T$ at a vertex $r$ of maximum eccentricity $\operatorname{diam}(T)$. Let $t$ be a leaf at maximum distance from $r, v$ be the parent of $t$, and $u$ be the parent of $v$ in the rooted tree. If $\operatorname{diam}(T) \geq 4$, then let $w$ be the parent of $u$. If $\operatorname{diam}(T) \geq 5$, then let $d$ be the parent of $w$. If $\operatorname{diam}(T) \geq 6$, then let $e$ be the parent of $d$. By $T_{x}$ we denote the subtree induced by a vertex $x$ and its descendants in the rooted tree $T$.

Assume that among the children of $u$ there is a support vertex $x$, other than $v$. Let $y$ be the leaf adjacent to $x$. Let $T^{\prime}=T-T_{v}$. Let $D$ be a $\gamma_{c t d}(T)$-set. By observation 1 , the vertices $y, t \in D$. To dominate $u$ either 
$v$ or $x$ belongs to $D$. If $x \in D$ then $D \backslash\{t\}$ is a CTDS of the tree $T^{\prime}$. Now assume that $v \in D$. Then $(D \backslash\{v, t\}) \cup\{x\}$ is a CTDS of the tree $T^{\prime}$. Thus $\gamma_{c t d}\left(T^{\prime}\right) \leq \gamma_{c t d}(T)-1$. Let $D^{\prime}$ be a $\gamma\left(T^{\prime}\right)$-set. To dominate $y, u$ the vertex $x \in D^{\prime}$. It is easy to see that $D^{\prime} \cup\{v\}$ is a DS of the tree $T$. Thus $\gamma(T) \leq \gamma\left(T^{\prime}\right)+1$. We get $\gamma_{c t d}\left(T^{\prime}\right) \leq \gamma_{c t d}(T)-1=\gamma(T)-1 \leq \gamma\left(T^{\prime}\right)$. This implies that $\gamma_{c t d}\left(T^{\prime}\right)=\gamma\left(T^{\prime}\right)$. By the inductive hypothesis $T^{\prime} \in T$. The tree $T$ is obtained from $T^{\prime}$ by operation $O_{1}$. Thus $T \in T$.

Assume that some child of $u$, say $x$, is a leaf. Let $T^{\prime}=T-T_{v}$. Let $D$ be a $\gamma_{c t d}(T)$-set. By observation 1 , the vertices $x, t \in D$. It is easy to see that $(D \backslash\{t\})$ is a CTDS of the tree $T^{\prime}$. Thus $\gamma_{c t d}\left(T^{\prime}\right) \leq \gamma_{c t d}(T)-1$. Let $D^{\prime}$ be a $\gamma\left(T^{\prime}\right)$-set. To dominate $x$ the vertex $u \in D^{\prime}$. It is easy to see that $D^{\prime} \cup\{v\}$ is a DS of the tree $T$. Thus $\gamma(T) \leq \gamma\left(T^{\prime}\right)+1$. We get $\gamma_{c t d}\left(T^{\prime}\right) \leq \gamma_{c t d}(T)-1=\gamma(T)-1 \leq \gamma\left(T^{\prime}\right)$. This implies that $\gamma_{c t d}\left(T^{\prime}\right)=\gamma\left(T^{\prime}\right)$. By the inductive hypothesis $T^{\prime} \in T$. The tree $T$ is obtained from $T^{\prime}$ by operation $O_{2}$. Thus $T \in T$.

Assume that $d_{T}(u)=2$. First assume that $d_{T}(w) \geq 3$. Let $T^{\prime}=T-T_{u}$. Let $D^{\prime}$ be a $\gamma\left(T^{\prime}\right)$-set. It is easy to see that $D^{\prime} \cup\{v\}$ is a DS of the tree $T$. Thus $\gamma(T) \leq \gamma\left(T^{\prime}\right)+1$. Let $D$ be a $\gamma_{c t d}(T)$-set. By observation $1, t \in D$. Since the induced sub graph of $V \backslash D$ is a tree, the vertex $v \in D$. It is clear that $w$ is dominated by a vertex adjacent to it other than $u$. We have $D \backslash\{v, t\}$ is a CTDS of the tree $T^{\prime}$. Thus $\gamma_{c t d}\left(T^{\prime}\right) \leq \gamma_{c t d}(T)-2$. We now get $\gamma_{c t d}\left(T^{\prime}\right) \leq \gamma_{c t d}(T)-2=\gamma(T)-2 \leq \gamma\left(T^{\prime}\right)-1<\gamma\left(T^{\prime}\right)$.

Assume that $d_{T}(w)=1$. We have $T=P_{4}$. This gives $\gamma_{c t d}(T)=2=$ $\gamma(T)$. Thus $T \in T$. Now assume that $d_{T}(w)=2$. Let $T^{\prime}=T-T_{u}$. Let $D^{\prime}$ be a $\gamma\left(T^{\prime}\right)$-set. It is easy to see that $D^{\prime} \cup\{v\}$ is a DS of the tree $T$. Thus $\gamma(T) \leq \gamma\left(T^{\prime}\right)+1$. Let $D$ be a $\gamma_{c t d}(T)$-set. The induced sub graph $\langle V \backslash D\rangle$ is a tree therefore, we have $t, v, u, w \in D$. It is obvious that $D \backslash\{v, u, t\}$ is a CTDS of the tree $T^{\prime}$. Thus $\gamma_{c t d}\left(T^{\prime}\right) \leq \gamma_{c t d}(T)-3$. We get $\gamma_{c t d}\left(T^{\prime}\right) \leq \gamma_{c t d}(T)-3=\gamma(T)-3 \leq \gamma\left(T^{\prime}\right)-2<\gamma\left(T^{\prime}\right)$.

As an immediate consequence of Lemmas 1 and 2, we have the following characterization of the trees with equal domination and complementary tree domination numbers.

Theorem 3. Let $T$ be a tree. Then $\gamma_{c t d}(T)=\gamma(T)$ if and only if $T \in T$.

\section{Complementary tree domination and edge-vertex domina- tion numbers of trees}

We start this section by proving that for any tree $T, \gamma_{c t d}(T)>\gamma_{e v}(T)$. 
Lemma 4. For every tree $T$ we have $\gamma_{c t d}(T)>\gamma_{e v}(T)$.

Proof. If $\operatorname{diam}(T) \leq 3$, then we get $\gamma_{e v}(T)=1<2 \leq \gamma_{c t d}(T)$. Now assume that $\operatorname{diam}(T) \geq 4$. Thus the order $n$ of the tree $T$ is at least five. The result we obtain by the induction on the number $n$. Assume that the lemma is true for every tree $T^{\prime}$ of order $n^{\prime}<n$.

First assume that some support vertex of $T$, say $x$, is strong. Let $y$ be a leaf adjacent to $x$. Let $T^{\prime}=T-y$. Let $D^{\prime}$ be any $\gamma_{e v}\left(T^{\prime}\right)$-set. It is easy to see that $D^{\prime}$ is an EVDS of the tree $T$. Thus $\gamma_{e v}(T) \leq \gamma_{e v}\left(T^{\prime}\right)$. Let $D$ be a $\gamma_{c t d}(T)$-set. It is obvious that $D \backslash\{t\}$ is a CTDS of the tree $T^{\prime}$. Thus $\gamma_{c t d}\left(T^{\prime}\right) \leq \gamma_{c t d}(T)-1$. We now get $\gamma_{c t d}(T) \geq \gamma_{c t d}\left(T^{\prime}\right)+1>\gamma_{e v}\left(T^{\prime}\right)+1 \geq$ $\gamma_{e v}(T)+1>\gamma_{e v}(T)$.

We now root $T$ at a vertex $r$ of maximum eccentricity $\operatorname{diam}(T)$. Let $t$ be a leaf at maximum distance from $r, v$ be the parent of $t, u$ be the parent of $v$, and $w$ be the parent of $u$ in the rooted tree. By $T_{x}$ we denote the subtree induced by a vertex $x$ and its descendants in the rooted tree $T$.

Assume that some child of $u$, say $x$, is a leaf. Let $T^{\prime}=T-T_{v}$. Let $D^{\prime}$ be a $\gamma_{e v}\left(T^{\prime}\right)$-set. It is obvious that $D \backslash\{u v\}$ is an EVDS of the tree $T$. Thus $\gamma_{e v}(T) \leq \gamma_{e v}\left(T^{\prime}\right)+1$. Let $D$ be a $\gamma_{c t d}(T)$-set. Obviously $D \backslash\{t, v\}$ is a CTDS of the tree $T^{\prime}$. Thus $\gamma_{c t d}\left(T^{\prime}\right) \leq \gamma_{c t d}(T)-2$. We now get $\gamma_{c t d}(T) \geq \gamma_{c t d}\left(T^{\prime}\right)+2>\gamma_{e v}\left(T^{\prime}\right)+2 \geq \gamma_{e v}(T)+1>\gamma_{e v}(T)$.

Assume that among the children of $u$ there is a support vertex, say $x$, other than $v$. Let $T^{\prime}=T-T_{v}$. Let $D^{\prime}$ be a $\gamma_{e v}\left(T^{\prime}\right)$-set. It is obvious that $D \backslash\{u v\}$ is an EVDS of the tree $T$. Thus $\gamma_{e v}(T) \leq \gamma_{e v}\left(T^{\prime}\right)+1$. Let $D$ be a $\gamma_{c t d}(T)$-set. Obviously $D \backslash\{t, v\}$ is a CTDS of the tree $T^{\prime}$. Thus $\gamma_{c t d}\left(T^{\prime}\right) \leq \gamma_{c t d}(T)-2$. We now get $\gamma_{c t d}(T) \geq \gamma_{c t d}\left(T^{\prime}\right)+2>\gamma_{e v}\left(T^{\prime}\right)+2 \geq$ $\gamma_{e v}(T)+1>\gamma_{e v}(T)$.

Assume that $d_{T}(u)=2$. Let $T^{\prime}=T-T_{u}$. Let $D^{\prime}$ be a $\gamma_{e v}\left(T^{\prime}\right)$-set. It is obvious that $D \backslash\{u v\}$ is an EVDS of the tree $T$. Thus $\gamma_{e v}(T) \leq \gamma_{e v}\left(T^{\prime}\right)+1$. Let $D$ be a $\gamma_{c t d}(T)$-set. Let $k$ be a neighbor of $w$. If $k \in D$, the $D \backslash\{t, v\}$ is a CTDS of the tree $T^{\prime}$. If $k \notin D$, the set $(D \backslash\{u, v, t\}) \cup\{k\}$ is a CTDS of the tree $T^{\prime}$. Thus $\gamma_{c t d}\left(T^{\prime}\right) \leq \gamma_{c t d}(T)-2$. We now get $\gamma_{c t d}(T) \geq \gamma_{c t d}\left(T^{\prime}\right)+2>$ $\gamma_{e v}\left(T^{\prime}\right)+2 \geq \gamma_{e v}(T)+1>\gamma_{e v}(T)$.

We characterize all trees with complementary tree domination number equal to $e v$-domination number plus one. For this purpose we introduce a family $F$ of trees $T=T_{k}$ that can be obtained as follows. Let $T_{1} \in\left\{P_{3}, P_{4}\right\}$. If $k$ is a positive integer, then $T_{k+1}$ can be obtained recursively from $T_{k}$ by one of the following operations. 
- Operation $O_{1}$ : Attach a path $P_{2}$ by joining its any vertex to a vertex of $T_{k} \neq P_{3}$, which is not a leaf and is adjacent to a support vertex of degree two.

Now we prove that for every tree of the family $F$, the complementary tree domination number is equal to $e v$-domination number plus one.

Lemma 5. If $T \in F$, then $\gamma_{c t d}(T)=\gamma_{e v}(T)+1$.

Proof. We use the induction on the number $k$ of operations performed to construct the tree. If $T=T_{1}=P_{3}$, then obviously $\gamma_{c t d}(T)=2=1+1=$ $\gamma_{e v}(T)+1$. If $T=T_{1}=P_{4}$, then obviously $\gamma_{c t d}(T)=2=1+1=\gamma_{e v}(T)+1$. Let $k \geq 2$ be an integer. Assume that the result is true for every $T^{\prime}=T_{k}$ of the family $F$ constructed by $k$ operations.

First assume that $T$ is obtained from $T^{\prime}$ by operation $O_{1}$. The vertex to which is attached $P_{2}$ we denote by $x$. Let $u_{1} u_{2}$ mean the attached path. Let $u_{1}$ be joined to $x$. The path $P_{2}$ adjacent to $x$ and different from $u_{1} u_{2}$ we denote by $v_{1} v_{2}$. Let $v_{1}$ be adjacent to $x$. Let $D^{\prime}$ be a $\gamma_{c t d}\left(T^{\prime}\right)$-set. The vertex $v_{2} \in D^{\prime}$. It is obvious that $D^{\prime} \cup\left\{u_{2}\right\}$ is a CTDS of a tree $T$. Thus $\gamma_{c t d}(T) \leq \gamma_{c t d}\left(T^{\prime}\right)+1$. Let $D$ be a $\gamma_{e v}(T)$-set. To dominate $u_{2}$ and $v_{2}$, the edges $x u_{1}, x v_{1} \in D$. It is clear that $D \backslash\left\{x u_{1}\right\}$ is an EVDS of the tree $T^{\prime}$. Thus $\gamma_{e v}\left(T^{\prime}\right) \leq \gamma_{e v}(T)-1$. Now we get $\gamma_{c t d}(T) \leq \gamma_{c t d}\left(T^{\prime}\right)+1=$ $\gamma_{e v}\left(T^{\prime}\right)+1+1 \leq \gamma_{e v}(T)+1$. On the other hand, by lemma 4 , we have $\gamma_{c t d}(T) \geq \gamma_{e v}(T)+1$. This implies that $\gamma_{c t d}(T)=\gamma_{e v}(T)+1$.

Lemma 6. Let $T$ be a tree of order $n \geq 3$. If $\gamma_{c t d}(T)=\gamma_{e v}(T)+1$, then $T \in F$.

Proof. If $\operatorname{diam}(T)=2$, then $T$ is a star. If $T=P_{3}$, then $T \in F$. If $T$ is a star different from $P_{3}$, then $\gamma_{c t d}(T)=n-1>2=\gamma_{e v}(T)+1$. Now assume that $\operatorname{diam}(T) \geq 3$. Thus the order $n$ of the tree $T$ is at least four. The result we obtain by the induction on the number $n$. Assume that the theorem is true for every tree $T^{\prime}$ or order $n^{\prime}<n$.

First assume that some support vertex of $T$, say $u$, is strong. Let $y$ be a leaf adjacent to $x$. Let $T^{\prime}=T-y$. Let $D^{\prime}$ be any $\gamma_{e v}\left(T^{\prime}\right)$-set. It is easy to see that $D^{\prime}$ is an EVDS of the tree $T$. Thus $\gamma_{e v}(T) \leq \gamma_{e v}\left(T^{\prime}\right)$. Let $D$ be a $\gamma_{c t d}(T)$-set. By observation 1 , the leaf $y \in D$. It is easy to see that $D \backslash\{y\}$ is an CTDS of the tree $T^{\prime}$. Thus $\gamma_{c t d}\left(T^{\prime}\right) \leq \gamma_{c t d}(T)-1$. We now get $\gamma_{c t d}\left(T^{\prime}\right) \leq \gamma_{c t d}(T)-1=\gamma_{e v}(T)+1-1 \leq \gamma_{e v}\left(T^{\prime}\right)<\gamma_{e v}\left(T^{\prime}\right)+1$. 
We now root $T$ at a vertex $r$ of maximum eccentricity $\operatorname{diam}(T)$. Let $t$ be a leaf at maximum distance from $r, v$ be the parent of $t$, and $u$ be the parent of $v$ in the rooted tree. If $\operatorname{diam}(T) \geq 4$, then let $w$ be the parent of $u$. If $\operatorname{diam}(T) \geq 5$, then let $d$ be the parent of $w$. By $T_{x}$ we denote the subtree induced by a vertex $x$ and its descendants in the rooted tree $T$.

Assume that among the children of $u$ there is a support vertex, say $x$, other than $v$. Let $y$ be a leaf adjacent to $x$. Let $T^{\prime}=T-T_{v}$. Let $D^{\prime}$ be any $\gamma_{e v}\left(T^{\prime}\right)$-set. It is easy to see that $D^{\prime} \cup\{u v\}$ is an EVDS of the tree $T$. Thus $\gamma_{e v}(T) \leq \gamma_{e v}\left(T^{\prime}\right)+1$. Now let $D$ be a $\gamma_{c t d}(T)$-set. By observation 1 , the leaves $y, t \in D$. If $v \in D$, then clearly $(D \backslash\{v, t\}) \cup\{x\}$ is a CTDS of the tree $T^{\prime}$. If $v \notin D$, then $D \backslash\{t\}$ is a CTDS of the tree $T^{\prime}$. Thus $\gamma_{c t d}\left(T^{\prime}\right) \leq$ $\gamma_{c t d}(T)-1$. We now get $\gamma_{c t d}\left(T^{\prime}\right) \leq \gamma_{c t d}(T)-1=\gamma_{e v}(T)+1-1 \leq \gamma_{e v}\left(T^{\prime}\right)+1$. On the other hand by Lemma 4 we have $\gamma_{c t d}\left(T^{\prime}\right) \geq \gamma_{e v}\left(T^{\prime}\right)+1$. This implies that $\gamma_{c t d}\left(T^{\prime}\right)=\gamma_{e v}\left(T^{\prime}\right)+1$. By the inductive hypothesis we have $T^{\prime} \in F$. The tree $T$ can be obtained from $T^{\prime}$ by operation $O_{1}$. Thus $T \in T$.

Assume that some child of $u$, say $x$, is a leaf. Let $T^{\prime}=T-T_{v}$. Let $D^{\prime}$ be a $\gamma_{e v}\left(T^{\prime}\right)$-set. It is easy to see that $D^{\prime} \cup\{u v\}$ is an EVDS of the tree $T$. Thus $\gamma_{e v}(T) \leq \gamma_{e v}\left(T^{\prime}\right)+1$. Let $D$ be a $\gamma_{c t d}(T)$-set. Clearly $t, x, v \in D$. It is obvious that $D \backslash\{t, v\}$ is a CTDS of the tree $T^{\prime}$. Thus $\gamma_{c t d}\left(T^{\prime}\right) \leq \gamma_{c t d}(T)-2$. We now get $\gamma_{c t d}\left(T^{\prime}\right) \leq \gamma_{c t d}(T)-2=\gamma_{e v}(T)+1-2 \leq \gamma_{e v}\left(T^{\prime}\right)<\gamma_{e v}\left(T^{\prime}\right)+1$.

Now assume that $d_{T}(u)=2$. If $d_{T}(w)=1$, then $T=P_{4} \in F$. Now assume that $d_{T}(w) \geq 3$. Assume that there is a child of $w$ other than $u$, say $x$, such that the distance of $w$ to the most distant vertex of $T_{k}$ is three or two or one. It suffices to consider the case $T_{k}$ is $P_{3}$ or $P_{2}$ or $P_{1}$. Let $T^{\prime}=T-T_{u}$. Let $D^{\prime}$ be a $\gamma_{e v}\left(T^{\prime}\right)$-set. It is easy to see that $D^{\prime} \cup\{u v\}$ is an EVDS of the tree $T$. Thus $\gamma_{e v}(T) \leq \gamma_{e v}\left(T^{\prime}\right)+1$. There exists a $\gamma_{c t d}(T)$-set that does not contain the vertex $u$. Let $D$ be such a set. It is obvious that $t, v \in D$. It is easy to see that $D \backslash\{t, v\}$ is a CTDS of the tree $T^{\prime}$. Thus $\gamma_{c t d}\left(T^{\prime}\right) \leq \gamma_{c t d}(T)-2$. We now get $\gamma_{c t d}\left(T^{\prime}\right) \leq \gamma_{c t d}(T)-2=\gamma_{e v}(T)+1-2 \leq$ $\gamma_{e v}\left(T^{\prime}\right)<\gamma_{e v}\left(T^{\prime}\right)+1$.

Now assume that $d_{T}(w)=2$. If $d_{T}(d)=1$, then $T=P_{5}$. We have $\gamma_{c t d}(T)=4=3+1>\gamma_{e v}(T)=1$. Assume that $d_{T}(e) \geq 2$. Let $T^{\prime}=T-T_{u}$. Let $D^{\prime}$ be a $\gamma_{e v}\left(T^{\prime}\right)$-set. It is easy to see that $D^{\prime} \cup\{u v\}$ is an EVDS of the tree $T$. Thus $\gamma_{e v}(T) \leq \gamma_{e v}\left(T^{\prime}\right)+1$. Let $D$ be such a $\gamma_{c t d}(T)$ set. It is obvious that $t, v, u, w \in D$. It is easy to see that $D \backslash\{t, v, u\}$ is a CTDS of the tree $T^{\prime}$. Thus $\gamma_{c t d}\left(T^{\prime}\right) \leq \gamma_{c t d}(T)-3$. We now get $\gamma_{c t d}\left(T^{\prime}\right) \leq \gamma_{c t d}(T)-3=\gamma_{e v}(T)+1-3 \leq \gamma_{e v}\left(T^{\prime}\right)-1<\gamma_{e v}\left(T^{\prime}\right)+1$. 
As an immediate consequence of Lemmas 5 and 6, we have the following characterization of the trees with complementary tree domination number and edge-vertex domination number plus one.

Theorem 7. Let $T$ be a tree. Then $\gamma_{c t d}(T)=\gamma_{e v}(T)+1$ if and only if $T \in F$.

\title{
References
}

[1] T. Haynes, S. Hedetniemi and P. Slater, Fundamentals of Domination in Graphs, Marcel Dekker, New York, (1998).

[2] T. Haynes, S. Hedetniemi and P. Slater (eds.), Domination in Graphs: Advanced Topics, Marcel Dekker, New York, (1998).

[3] X. Hou, A characterization of trees with equal domination and total domination numbers, Ars Combinatoria 97A, pp. 499-508, (2010).

[4] M. Krzywkowski, On trees with double domination number equal to 2-domination number plus one, Houston Journal of Mathematics 39, pp. 427-440, (2013).

[5] S. Muthammai, M. Bhanumathi and P. Vidhya, Complementary tree Domination number of a graph, International Mathematical Forum 6, pp. 1273-1282, (2011).

[6] J. Peters, Theoretical and Algorithmic Results on Domination and connectivity, Ph. D. Thesis, Clemson University, (1986).

\author{
B. Krishnakumari \\ Department of Mathematics \\ School of Humanities and Sciences \\ SASTRA University, \\ Thanjavur-613 401, \\ Tamilnadu, \\ India \\ e-mail : krishnakumari@maths.sastra.edu \\ and
}




\section{Y. B. Venkatakrishnan}

Department of Mathematics

School of Humanities and Sciences

SASTRA University,

Thanjavur-613 401,

Tamilnadu,

India

e-mail : ybvenkatakrishnan2@gmail.com 